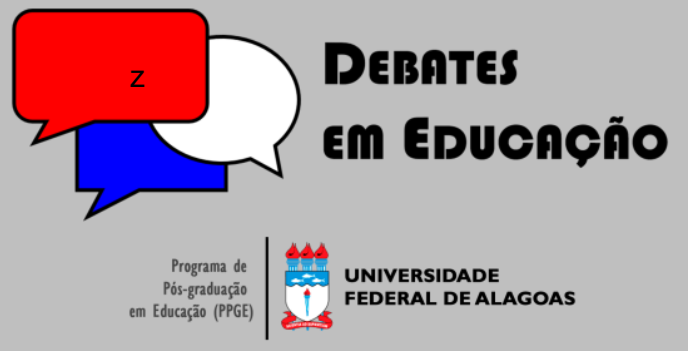

ISSN Eletrônico 2175-6600

Vol. 10 | No. 22 | Set./Dez. | 2018

Teresa Cardoso 9 in

Universidade Aberta (UAb - Portugal) tcardoso.uab@gmail.com

Teresa Coimbra

Universidade Aberta (UAb - Portugal)

tcoimbra@lead.uab.pt

Artur Mateus

Universidade Aberta (UAb - Portugal)

artur.mateus@ipleiria.pt

\section{ANÁLISE MATEMÁTICA E REALIDADE AUMENTADA: UM ESTUDO NO ENSINO SUPERIOR EM PORTUGAL}

\section{RESUMO}

Neste texto, começamos por definir Realidade Aumentada (RA), enquadrando-a nas tecnologias tridimensionais, referindo a sua importância e atualidade. Depois, num breve estado da arte, apresentamos um mapeamento, a partir de estudos que focam a RA em contextos educacionais, nomeadamente no ensino superior e no domínio da matemática. Posteriormente, descrevemos parte do estudo desenvolvido no âmbito do trabalho de doutoramento "As Tecnologias Tridimensionais como contributo para a aprendizagem da Matemática no Ensino Superior", que teve como objetivo principal contribuir para uma compreensão mais profunda de como as tecnologias tridimensionais podem potenciar o ensino e a aprendizagem da Matemática, no contexto nacional do ensino superior, em particular na área das engenharias. Da análise dos nossos dados e resultados, foi possível concluir que os desafios que se colocam hoje ao nível dos métodos de ensino, da aquisição e consequente consolidação do conhecimento podem ser em parte (cor)respondidos com a aplicação de tecnologias disponíveis, como a RA, que permitam uma compreensão mais completa dos conteúdos, levando à endogeneização de conhecimento e também à internalização de competências com bases mais sólidas.

Palavras-chave: Realidade Aumentada. Conteúdos tridimensionais. Tecnologias da informação e comunicação (TIC). Estudantes de ensino superior. Ensino e aprendizagem da matemática.

\section{MATHEMATICAL ANALYSIS AND AUGMENTED REALITY: A STUDY IN HIGHER EDUCATION IN PORTUGAL}

\begin{abstract}
In this text, we begin by defining Augmented Reality (AR), framing it in three-dimensional technologies, referring to its importance and contemporaneity. Then, in a brief state of the art, we present a mapping of studies focusing on AR in educational contexts, namely in higher education and mathematics. Subsequently, we describe part of the study developed in the scope of the doctoral work "The Three Dimensional Technologies as a contribution to the learning of Mathematics in Higher Education", whose main objective was to contribute to a deeper understanding of how three-dimensional technologies can enhance the teaching and learning of Mathematics, in the national context of higher education, particularly in the area of engineering. From the analysis of the our data and results, it was possible to conclude that the challenges that are posed today in the teaching methods, acquisition and consequent consolidation of knowledge can be partly answered with the application of available technologies, such as AR, which allow a more complete understanding of the contents, leading to a endogenization of knowledge and internalization of competences with more solid bases.
\end{abstract}

Keywords: Augmented Reality. Three-dimensional Contents. Information and communications technology (ICT). Higher education students. Teaching and learning of mathematics.

Submetido em: 18/07/2018

Aceito em: 21/10/2018

Publicado em: 21/12/2018

DOI: 10.28998/2175-6600.2018v10n22p271-283 


\section{INTRODUÇÃO}

O termo Realidade Aumentada (RA) foi cunhado por Tom Caudell, em 1990, enquanto trabalhava na Boing (VAUGHAN-NICHOLS, 2009) e traduz a integração de imagens virtuais no mundo real, ou seja, a realidade é aumentada de elementos virtuais (MILGRAM, 2006; KESIM \& OZARSLAN, 2012; WU et al., 2013). A integração daquele tipo de imagens é efetuada através da utilização das tecnologias de informação e comunicação (TIC), através de um dispositivo móvel com uma câmara (computador, tablet, telemóvel com sistema operativo android ou IOS) que permite aceder a conteúdos disponibilizados com RA. Por sua vez, o desenvolvimento de tais conteúdos potencia uma maior autonomia na aprendizagem e a utilização de sistemas que suportam o mobile-learning (ISMAIL et al., 2010). Além disso, a própria exploração das TIC pelos estudantes poderá promover competências de colaboração, inovação e criatividade (REDECKER, 2008).

Uma característica que as aplicações em RA proporcionam é a integração e interação entre o real e o virtual, permitindo desta forma uma grande versatilidade e criatividade nas aplicações. Por exemplo, a RA permite que se desenvolvam conteúdos como livros, sebentas ou apresentações, de forma convencional, em papel, acrescentando, no entanto, elementos gráficos que uma aplicação de RA reconheça e que tenham sido programados para que, quando visualizados, ativem elementos adicionais de explicação (por exemplo: ficheiros tridimensionais, vídeos explicativos e/ou imagens). Ou seja, a RA permite adicionar informação digital ao mundo real, não somente ao que está definido em duas dimensões (2D), como o caso da inclusão de camadas de conteúdos tridimensionais virtuais sobre conteúdos de papel físico (papel 2D), mas também incluir informação digital e virtual sobre objetos tridimensionais físicos. Esses podem ser feitos por impressão 3D, e, nesse caso, a perceção virtual pode ser complementada pelo toque tangível. Dito de outro modo: estende-se a perceção virtual à perceção física tangível.

Esse formato de conteúdos pode ser adaptado à aprendizagem a distância, quer formal, quer informal, já que conteúdos preparados sobre temas visualizados em contexto real podem ser acedidos por qualquer indivíduo a qualquer altura. Por exemplo, a preparação de conteúdos no domínio da biologia pode permitir que numa exploração de campo o sistema móvel (baseado nas TIC, telemóvel ou tablet) identifique um determinado tipo de planta e forneça informação técnica/científica sobre a mesma in situ e em tempo real. Dessa forma, a exploração de campo acompanhada por informação detalhada potencia a internalização do conhecimento. Pode-se, então, dizer que as tecnologias de RA permitem integrar o conhecimento teórico em contextos reais e também permitem integrar 
contextos reais em formas de apresentação mais teóricas. Da aproximação/integração dos dois formatos de informação advêm importantes vantagens, nomeadamente porque é possível materializar conceitos, não perdendo o rigor inerente aos mesmos, e também potenciar a perceção de conceitos mais abstratos.

As áreas de estudo e aprendizagem para as quais a RA pode ser útil são diversas e representam uma mais-valia significativa naquelas que exigem mais interação prática e experimental, como é o caso dos cursos de engenharia e ciências (LIAROKAPIS et al., 2004; NEE et al., 2012). Também se podem fazer conteúdos conjugando diversos outros ambientes, e não só em sala de aula. Assim, a RA permite o desenvolvimento de conteúdos aos quais cada indivíduo pode aceder em diversos contextos/ambientes, além dos mais comuns (como em casa, num escritório, numa sala de aula). E, portanto, potencia a interação entre a observação in situ do mundo real e a adição de conteúdos teóricos e explicativos (por exemplo, o EcoMobile, descrito por KAMARAINEN et al., 2013).

Essa flexibilidade que as ferramentas de RA oferecem permite uma maior experimentação e exploração do real, com a introdução de explicações virtuais em tempo real. De fato, os conteúdos em RA permitem que os estudantes, quando expostos a situações reais, interpretem com maior flexibilidade e obtenham explicações sobre a observação real, efetuada em tempo real. Desse modo, a aprendizagem centra-se no indivíduo e cada um pode ter acesso a explicações e a apoio sob a forma de conteúdos de RA, sendo estes diferenciados dos demais, e, no momento em que a aprendizagem se processa. A RA tem vido a ser aplicada de forma progressiva no domínio da educação, dando vida por exemplo aos livros (BILLINGHURSTA et al., 2001; KAUFMANN \& SCHMALSTIEG, 2003). Justifica-se, pois, que se descreva a evolução da RA e da sua aplicação à educação, o que faremos no ponto seguinte, focalizando no domínio da matemática.

\section{REVISÃO BIBLIOGRÁFICA}

As tecnologias de RA estão estreitamente ligadas à capacidade e ao cálculo computacional e, dessa forma, a sua disponibilização está relacionada com o trajeto do desenvolvimento dos computadores pessoais. Assim, importa começar por abordar alguns dos trabalhos desenvolvidos em nível internacional e nacional, pela aplicação dessas tecnologias, principalmente no domínio da educação e do ensino.

Relativamente às tecnologias tridimensionais, e apesar de a sua aplicação no ensino/aprendizagem ainda não ser massiva, são diversas as áreas de conhecimento em 
que têm vindo a ser implementadas e estudadas, por diversos autores (entre outros: FONSECA et al., 2013; KAMARAINEN et al., 2013; WU et al., 2013; MARTIN-GUTIERREZ et al., 2012; NEE et al., 2012; KAUFMANN \& SCHMALSTIEG, 2003). Assim, para poder ter uma perspetiva histórica e mais alargada da aplicação de conteúdos e tecnologias de RA no domínio da educação, formação e ensino, revisitamos alguns estudos e aplicações dos últimos anos.

Em 1997, Inkpen apresentou um estudo em que foram desenvolvidos conteúdos específicos para estimular a aprendizagem via computador. Estes conteúdos não foram desenvolvidos em RA. Contudo, foram precursores na análise do efeito da aprendizagem baseada em tecnologias. Além do desenvolvimento específico de aplicações e de software para estímulo da aprendizagem, o autor analisou a possibilidade de trabalho simultâneo com dois ratos nos computadores. Os resultados mostraram que a motivação e a aprendizagem foram incrementadas com o trabalho em grupo, potencializada pela utilização simultânea de dois dispositivos de interação com o computador (ratos) em comparação com a utilização individual por cada criança.

No ano de 2001, e no domínio da matemática, Billinghursta e colaboradores desenvolveram conteúdos apresentados em RA, com o nome de “MagicBook". Já em 2003, e ainda no domínio da matemática, Kaufmann e Schmalstieg descrevem a implementação do sistema "Construct3D", que permitiu avaliar a importância e flexibilidade da RA, mesmo em ambientes colaborativos, e atestar o relevo desses ambientes na interação entre estudantes e entre estudantes e professor. Aquele sistema, constituído por conteúdos tridimensionais no domínio da matemática, apoiava-se em equipamentos de visualização e trabalho colaborativo, presencial ou remotamente (a distância). Em 2013, Salinas et. al. desenvolveram um software específico para modelação tridimensional de funções matemáticas e conduziram um estudo evidenciando o importante papel destas tecnologias na motivação de grupos e na potenciação do trabalho colaborativo.

Considerando outras áreas científicas, referimos que Liarokapis et. al. (2004) e Nee et al. (2012) estudaram a aplicação da RA no âmbito dos projetos e processos de fabrico, enquanto que Martín-Gutiérrez et al. (2012) estudaram a aplicação da RA na área da engenharia eletrotécnica e Fonseca et al. (2013) exploraram a sua aplicabilidade nos domínios da arquitetura.

Nos trabalhos analisados, verificaram-se vantagens na aplicação da RA. Por exemplo, no estudo de Martin-Gutierrez et al. (2012) foi sublinhado o aumento da capacidade de auto-aprendizagem dos estudantes, o que pode facultar ao docente mais tempo para explicar questões mais complexas. Já no estudo de Fonseca et al. (2013) 
referem-se as vantagens que as ferramentas de RA conferem no aumento da perceção espacial, a qual proporciona a visualização, in situ, de cenários hipotéticos para construção futura e, desse modo, uma exploração e análise de diversas soluções.

Num outro estudo anterior, sobre sistemas m-learning, Ismail e colaboradores (2010) constatam a manifesta satisfação por parte dos utilizadores em usar essas ferramentas de aprendizagem adicionais (de mobile learning), sentindo-se apoiados e motivados para a utilização de aplicativos móveis com uma linguagem acessível. De fato, os sistemas habitualmente usados em m-learning, tais como os referidos sistemas móveis de comunicação, podem, quando integrados com conteúdos de RA, potenciar as observações e explorações de campo porque se pode explicar a Realidade observada com a adição (Aumentada) de conteúdos virtuais adicionais (entre outros: vídeos explicativos, esquemas, desenhos tridimensionais). Essa interação contribui para uma maior autonomia no processo de aprendizagem. Na Figura 1, apresentamos um exemplo que desenvolvemos em RA para apoio ao ensino da matemática, e que incluímos no caderno-manual com exercícios de apoio.

Figura 1 - Representação 3D em RA de função matemática.

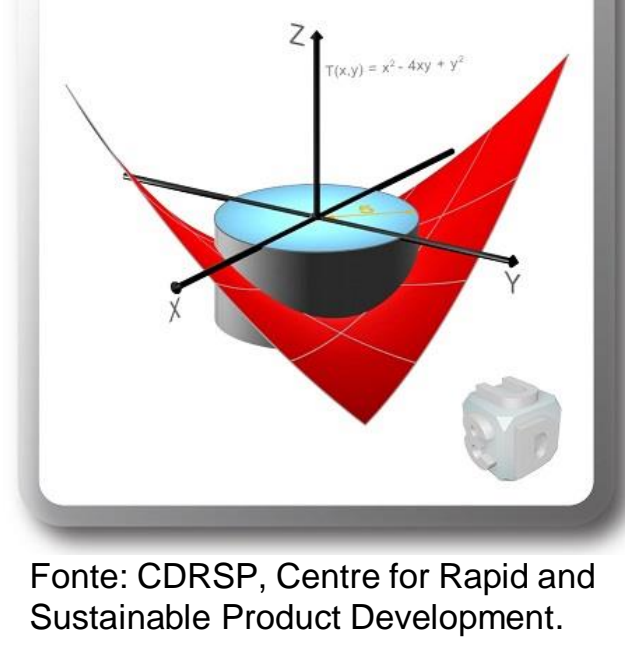

Os conteúdos de ensino/aprendizagem, nesse caso em particular da matemática, podem ser concebidos sob a forma mais comum, baseada numa explicação disponível em papel, que se complementa com a descrição de equações, baseada em figuras bidimensionais. A esses elementos podem ser acrescentados conteúdos como ficheiros tridimensionais, vídeos e explicações de passos intermédios. Faz-se, assim, uma integração entre um modo tradicional de visualização de conteúdos, através da sua consulta em papel (2D) e o recurso à tecnologia da RA (em 3D) que a complementa. Nos 
pontos seguintes, aprofundamos esse exemplo, que desenvolvemos em RA para apoio ao ensino da matemática e a sua implementação. A seguir, então, retomamos o nosso enquadramento metodológico, para depois discutirmos alguns dos resultados obtidos.

\section{METODOLOGIA}

Um dos fundamentos da nossa investigação foi compreender se e como determinadas inovações educacionais e tecnológicas são percecionadas e utilizadas. Assim, considerámos o Design-Based Research (DBR) como a metodologia mais adequada, já que essa visa descobrir as relações entre a teoria educacional, artefacto projetado e prática. No nosso estudo, participaram estudantes do Ensino Superior Politécnico, tendo sido feita uma triangulação de métodos (quantitativos e qualitativos) e implementadas técnicas de recolha e de análise de dados (respetivamente: inquérito por questionário e por entrevista, e observação; análise documental, análise estatística inferencial e descritiva, e análise de conteúdo). O DBR permite e encoraja múltiplas interações em todas as fases, resultando numa evolução contínua da intervenção, em que o conhecimento se constrói de forma circular ou espiralada (CARDOSO, 2007; CARDOSO et al., 2010).

Sendo o DBR relativamente recente e existindo diversos métodos e variações, podese afirmar que não há ainda consenso alargado sobre os procedimentos a observar nesse tipo de estudo. No entanto, adotamos as características essenciais à sua implementação, como especificamos a seguir, nomeadamente as seguintes etapas, que podem ocorrer simultaneamente ou até por uma ordem diferente: (i) formular um problema significativo; (ii) promover a colaboração entre investigadores; (iii) integrar conceitos teóricos relevantes sobre ensino e aprendizagem; (iv) conduzir uma revisão de literatura para sustentar as questões de investigação; (v) projetar uma intervenção educacional; (vi) desenvolver, implementar e rever o projeto; (vii) avaliar o impacto da intervenção do projeto; (viii) iterar o processo; (ix) escrever o relato do DBR.

A primeira etapa (i) foi encetada ainda numa fase embrionária dessa investigação e foi consolidada após uma consistente revisão da literatura (etapa iv). A segunda etapa (ii) incluiu a fase de pré-teste, quer aos conteúdos tridimensionais em RA por parte de alguns dos docentes do Instituto Politécnico de Leiria (IPL), quer ao inquérito por entrevista aos docentes - este inquérito e o respetivo guião foram discutidos e validados, simultaneamente por docentes de Análise Matemática e por investigadores especialistas, ambos constituindo um painel de peritos, de acordo com o método Delphi. A etapa (iii) está integrada na 
construção de toda a problemática apresentada e intimamente relacionada com a etapa (iv). A etapa (vi) foi iniciada aquando da construção dos conteúdos em RA e dos instrumentos de recolha de dados, nomeadamente, o inquérito por entrevista a docentes e o inquérito por questionário a estudantes. A etapa (vii) decorreu do segmento das anteriores e sempre que se justificou. A etapa final (ix) traduziu-se na tese que está disponível no repositório aberto da Universidade Aberta (COIMBRA, 2017).

A parte empírica do estudo foi desenvolvida também em várias fases, sendo a primeira o contacto com os atores diretamente implicados na utilização dos conteúdos 3D em RA, nomeadamente o Diretor da Escola Superior de Tecnologia e Educação do IPL, os coordenadores dos vários cursos de engenharia do IPL, o Coordenador do Departamento de Matemática, a Diretora da Unidade de Ensino a Distância do IPL, assim como os docentes de Análise Matémática (AM). Alguns desses docentes colaboraram diretamente na contrução dos conteúdos 3D em RA, tendo antes identificado os conteúdos científicos da unidade curricular (UC) mais pertinentes para a elaboração dos referidos conteúdos 3D em RA; os restantes docentes de AM foram informantes na realização do pré-teste ao inquérito por entrevista aos docentes. Essa fase teve várias interações que culminaram com a entrevista aos docentes e a implementação dos conteúdos 3D em RA em sala de aula, os quais foram sendo construídos (I), testados e corrigidos sistematicamente, com recurso a diversas aplicações e ferramentas - de programação com instruções em RA, de edição tridimensional (II), e de acesso (III), em fase de consulta (IV). A figura seguinte sistematiza as quatro fases que consideramos no desenvolvimento dos conteúdos $3 \mathrm{D}$ em RA que criamos, aplicados ao ensino da Matemática.

Figura 2 - Fases de edição dos conteúdos em RA.

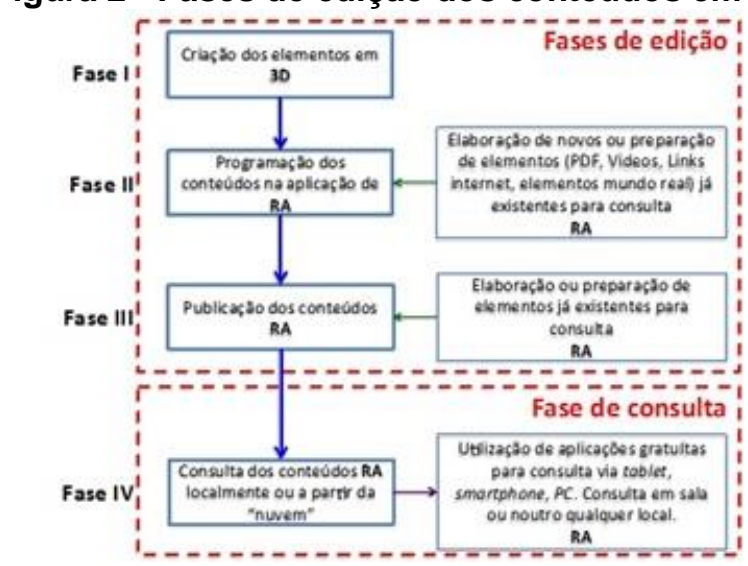

Fonte: Coimbra (2017, p. 97). 
As três primeiras fases (I, II e III) correspondem ao procedimento implementado com vistas à elaboração e preparação dos conteúdos para RA. Correspondem assim a uma etapa de edição. A fase IV corresponde à etapa de consulta por parte dos utilizadores.

Na edição, contempla-se o desenvolvimento de elementos 3D (fase I), que, no nosso caso, foram criados através da utilização de diferentes aplicações e software. Iniciamos a criação daqueles elementos com recurso a software de matemática com a capacidade de converter funções tridimensionais em ficheiros neutros, os quais contêm a descrição tridimensional das referidas funções. Assim, nessa fase e através da utilização das referidas aplicações de matemática, criaram-se vários ficheiros em formato neutro constituídos por malhas de polígonos definidas pela normal exterior. Os formatos experimentados foram: STL (Standard Tessellation Language), OBJ (desenvolvido pela Wavefront Technologies) e PLY (Polygon File Format). O STL traduz apenas a geometria enquanto que os restantes dois traduzem a geometria e a cor. Porém, foi selecionado o formato STL pela simplicidade da sua estrutura, a qual permite uma manipulação mais simples e eficaz (cf. figura 3).

Figura 3 - Estrutura de um ficheiro STL.

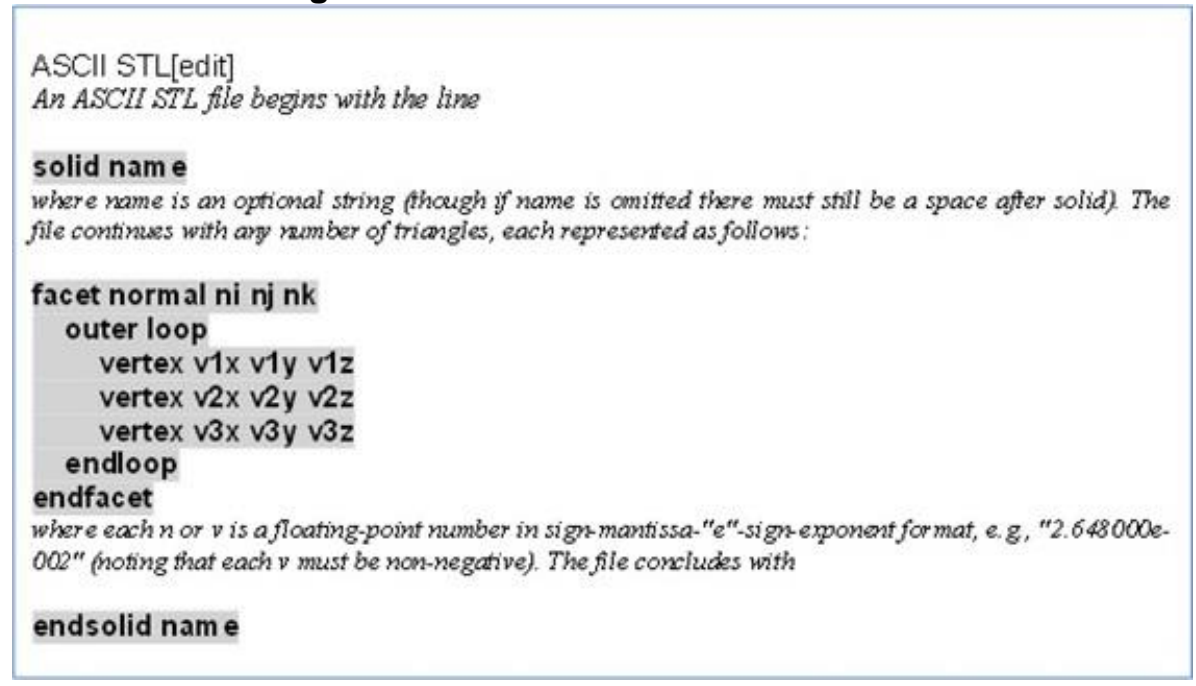

Fonte: CDRSP, Centre for Rapid and Sustainable Product Development.

É importante realçar que os conteúdos de AM selecionados para a construção dos exemplos do pré-teste, tiveram em consideração o fato de serem potencialmente melhorados com a tecnologia de $\mathrm{RA}$, já que têm uma componente geométrica associada à tridimensionalidade. Por outro lado, reportam-se a conceitos matemáticos onde os estudantes tendem geralmente a manifestar maiores dificuldades, como foi reconhecido pelos docentes que colaboraram conosco no estudo. A seguir retomamos a pergunta refletida no título deste texto para, com base numa síntese dos resultados da observação efetuada no pré-teste, tentarmos dar-Ihe resposta. 


\section{RESULTADOS E DISCUSSÕES}

No que diz respeito à aplicação em sala de aula dos conteúdos 3D em RA, que decorreu inicialmente numa fase de pré-teste no turno TP1 Pós-laboral de Engenharia Eletrotécnica do $2^{\circ}$ semestre, foram facultados 13 tablets e 13 manuais (Cadernos de Apoio), um por cada aluno. Estiveram presentes duas docentes, a docente titular do turno em questão e a docente da UC no $2 .^{\circ}$ semestre. Em sala de aula, foi possível registar imagens, interagir com os estudantes e observar algumas situações relevantes, de que são exemplo as figuras 4 e 5 .

Figuras 4 e 5: Pré-teste da aplicação dos conteúdos em RA em sala de aula
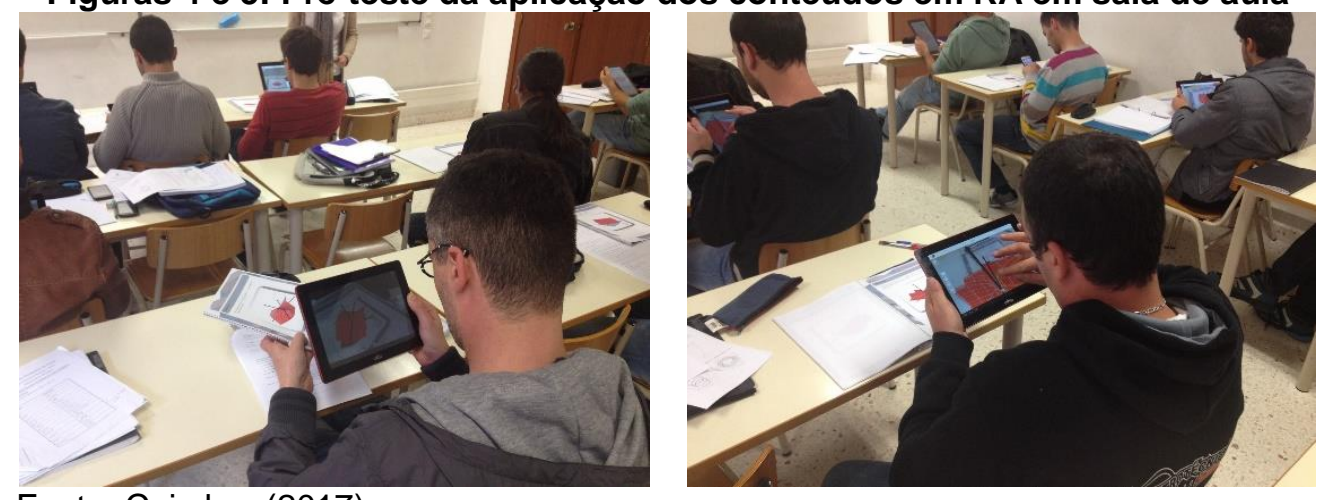

Fonte: Coimbra (2017).

Deste pré-teste e em relação aos tablets, salientamos que a sua utilização foi muito intuitiva, não tendo existido nenhum tipo de resistência. Surgiu, contudo, um dispositivo que não estava a aceder ao canal da aplicação Junaio, pelo que foi substituído por outro. No decorrer da interação, surgiu um outro dispositivo com falhas de comunicação, mas o estudante de forma autônoma optou por usar o seu telemóvel pessoal, tendo acedido rapidamente ao aplicativo e consequente canal de acesso.

Quanto aos conteúdos do programa curricular da UC, estes foram sendo apresentados progressivamente, com um caráter de revisão de conceitos, e os estudantes acompanharam atentamente todas as explicações verbais complementado-as com os conteúdos 3D em RA que tinham ao seu dispor. Constatamos que cerca de metade dos estudantes sentiu curiosidade em testar a nova abordagem dos conteúdos (em RA) com o recurso ao telemóvel pessoal, mesmo quando o tablet estava a funcionar em pleno; fizeramno de forma autônoma e bastante rápida.

Foram apresentados 7 exemplos dos 29 que o Caderno de Apoio apresenta para interação em 3D, tendo em conta os objetivos definidos para a referida aula. Em cada exemplo, foi possível interagir com o conteúdo, interpretar os resultados, quer numa 
perspetiva prática quer relacionando-os com os conceitos teóricos subjacentes. Essa interação demorou entre 20 e 30 minutos, incluindo o tempo de preparação dos dispositivos móveis e a respectiva explicação de utilização.

No final dessa sessão de pré-teste, 10 dos 13 estudantes responderam a um pequeno inquérito, que demorou cerca de 5 minutos. Os resultados mais relevantes no que à nossa investigação diz respeito são seguidamente sistematizados.

Apesar de a grande maioria dos estudantes não ter tido qualquer contacto prévio com os conteúdos, nem conhecer a RA, a não ser em contexto de jogo ou por divulgação na comunicação social, todos afirmaram que aceitariam com agrado a integração de conteúdos 3D na UC de AM sobretudo porque "facilita a aprendizagem" e por considerarem "mais perceptível" do que outras estratégias pedagógicas e abordagens tecnológicas. As questões que mais colocaram foram de cariz técnico, querendo saber mais acerca da tecnologia usada e da forma como foram construídos os conteúdos 3D, indagando acerca das potencialidades do software, demonstrando muita curiosidade e interesse.

Além disso, os estudantes chegaram a algumas conclusões importantes, como o fato de o dispositivo usado influenciar a forma de visualização dos conteúdos, quer pelo tamanho, quer pelo consumo de energia, ou mesmo pela captação de rede, a definição do ecrã do próprio equipamento, ou ainda pela luminosidade (quer do exterior, quer do dispositivo). Mesmo sendo a primeira vez que a grande maioria dos estudantes interagiu com essa tecnologia, foi bastante intuitiva a forma como decorreu. Não foram necessárias explicações muito detalhadas porque cada um foi descobrindo intuitivamente várias formas de manipular os exemplos apresentados. Foram muitos os estudantes que aproveitaram a aula do pré-teste para instalar a aplicação Junaio no seu telemóvel ou tablet, para poderem testar os conteúdos 3D em RA com os seus próprios equipamentos e noutros contextos que não apenas o da sala de aula.

Os estudantes revelaram muita autonomia na manipulação dos conteúdos, criaram formas adaptadas ao seu gosto pessoal para interagir com os dispositivos, testaram formas de visualização, trocaram ideias, gravaram vídeos do que estavam a visualizar e terem outra perspetiva dos conteúdos, e, no final da aula, referiram estar todos aptos para usar esta tecnologia sem qualquer dificuldade. Alguns dos comentários mais ouvidos foram: "As aulas deveriam ser todas assim"; "A melhor aula que já tive de Matemática”; "Dessa forma conseguimos visualizar melhor os conceitos e perceber melhor a teoria"; "Para ver gráficos em 3D isto é espetacular"; "Torna a matéria mais fácil de entender porque podemos visualizar melhor e interagir"; "Era bom podermos usar isso nas avaliações"; "Todas as aulas assim também poderia ser fator de distração, a não ser que o professor conseguisse 
controlar bem a aula". Portanto, das palavras dos estudantes inferimos que a grande maioria tem uma opinião favorável relativamente ao fato de a integração de conteúdos em RA poder promover a compreensão desses mesmos conteúdos e, desse modo, poder contribuir para melhorar a aprendizagem da matemática.

\section{CONCLUSÃO}

Pelo exposto, e ainda que se trate da parte do estudo que correspondeu ao 1. ciclo de iterações DBR, mais especificamente à fase de pré-teste dos conteúdos 3D criados para aulas de Análise Matemática, podemos afirmar que, nesse contexto particular, a RA permite melhorar a aprendizagem da matemática. No entanto, estamos cientes de que há aspectos a considerar para que esse potencial não seja comprometido. A título ilustrativo, e sintetizando, referimos que quando as condições de luminosidade são mais reduzidas, nem sempre é possível fazer a focagem automática, nem aceder com maior detalhe aos exemplos apresentados. Essas limitações devem ser acauteladas e ultrapassadas, para não condicionar uma melhor visualização dos conteúdos em RA.

A terminar, convocamos o ditado português que diz que "uma imagem vale mais do que mil palavras", e, se essa for uma imagem tridimensional então valerá ainda mais. Acreditamos, pois, pela nossa revisão da literatura e pela análise dos nossos próprios resultados, que uma imagem tridimensional imersa no mundo real valerá mais do que qualquer outra imagem (ou palavra). Dito de outro modo, e como reconheceram os estudantes, a RA torna os conceitos matemáticos mais fáceis de compreender porque permite visualizá-los melhor e interagir com eles. Assim, podemos desde já concluir que tecnologias tridimensionais como a RA potenciam o ensino e a aprendizagem da matemática no ensino superior.

\section{REFERÊNCIAS}

BILLINGHURSTA, M., KATOB, H. \& POUPYREV, I. The MagicBook: a transitional AR interface, Computers \& Graphics 25, Elsevier Science Ltd., p. 745-753, 2001.

CARDOSO, T. et al. MAECC®: um caminho para mapear investigação. Indagatio Didactica, 5(2) Tecnologias da Informação em Educação, 2013. Disponível em http://revistas.ua.pt/index.php/ID/article/view/2452/2323. Acesso em 18 jun.2018.

CARDOSO, T. et al. Revisão da Literatura e Sistematização do Conhecimento. Porto: Porto Editora, 2010. 
$\mathrm{xxx}$. As tecnologias tridimensionais como contributo para a aprendizagem da matemática no ensino superior (Tese de doutoramento). Lisboa: Universidade Aberta, 2017.

FONSECA, D. et al. Relationship between student profile, tool use, participation, and academic performance with the use of Augmented Reality technology for visualized architecture models. Computers in Human Behavior, 31, p. 434-445, 2013.

INKPEN, K. Adapting the Human-Computer Interface to Support Collaborative Learning Environments for Children (Tese de doutoramento). University of British Columbia:

Department of Computer Science, 1997.

ISMAIL, I., IDRUS, R. \& GUNASEGARAN, T. (2010). Motivation, Psychology and Language Effect on Mobile Learning in Universiti Sains Malaysia. Universiti Sains Malaysia, Penang, Malaysia.

KAMARAINEN, A. et al. (2013) EcoMOBILE: Integrating augmented reality and probeware with environmental education field trips. Computers \& Education, 68, p. 545-556, 2013.

KAUFMANN, H. \& SCHMALSTIEG, D. Mathematics and geometry education with collaborative augmented reality. Computers \& Graphics, 27, p. 339-345, 2003.

KESIM, M. \& OZARSLAN, Y. Augmented reality in education: current technologies and the potential for education. Procedia - Social and Behavioral Sciences, 47, p. 297302, 2012.

LIAROKAPIS, F., MOURKOUSSIS, N., WHITE, M., DARCY, J., SIFNIOTIS, M., PETRIDIS, P., BASU, A., LISTER, P. Web3D and augmented reality to support engineering education. World Transactions on Engineering and Technology Education, Vol.3, No.1, p. 11-14, 2004.

MARTIN-GUTIERREZ, J. et al. Improving strategy of self-learning in engineering: laboratories with augmented reality. Procedia - Social and Behavioral Sciences 5, p. 832-839, 2012.

MILGRAM, P. Some Human Factors Considerations for Designing Mixed Reality Interfaces. Virtual Media for Military Applications, Meeting Proceedings RTO-MP-HFM136, Keynote 1. Neuilly-sur-Seine, France: RTO, 2006. Disponível em http://www.rto.nato.int/abstracts.asp. Acesso em 18 jun.2018.

NEE, A. et al. Augmented reality applications in design and manufacturing. CIRP Annals - Manufacturing Technology, 61, p. 657-679, 2012.

REDECKER, C. Review of Learning 2.0 Practices: Study on the Impact of Web 2.0 Innovations on Education and Training in Europe. European Commission Joint Research Centre Institute for Prospective Technological Studies, 2008. Disponível em http://ftp.jrc.es/EURdoc/JRC49108.pdf. Acesso em 18 jun.2018.

SALINAS, P. et al. The Development of a Didactic Prototype for the Learning of Mathematics through Augmented Reality. 2013 International Conference on Virtual and Augmented Reality in Education. Procedia Computer Science, 25, p. 62-70, 2013.

VAUGHAN-NICHOLS, S. J., \& VAUGHAN-NICHOLS, S. J. Augmented reality: No Ionger a novelty? Computer, 42(12), 19-22, 2009. 
$\mathrm{WU}, \mathrm{H}$. et al. Current status, opportunities and challenges of augmented reality in education. Computers \& Education, 62, p. 41-49, 2013. 